\title{
Limitations of multidetector computed tomography angiography for the diagnosis of blunt cerebrovascular injury
}

\author{
Ramesh Grandhi, MD, ${ }^{1}$ Gregory M. Weiner, MD, ${ }^{2}$ Nitin Agarwal, MD, ${ }^{2}$ David M. Panczykowski, MD, ${ }^{2}$ \\ William J. Ares, MD, ${ }^{2}$ Jesse S. Rodriguez, PhD, ${ }^{1}$ Jonathan A. Gelfond, MD, PhD, ${ }^{3}$ \\ John G. Myers, MD, ${ }^{4}$ Louis H. Alarcon, MD, ${ }^{5}$ David O. Okonkwo, MD, PhD, ${ }^{2}$ and \\ Brian T. Jankowitz, MD²
}

\begin{abstract}
Departments of ${ }^{1}$ Neurosurgery, ${ }^{3}$ Epidemiology and Biostatistics, and ${ }^{4}$ Surgery, Division of Trauma and Emergency Surgery, University of Texas Health Science Center at San Antonio, Texas; and Departments of ${ }^{2}$ Neurological Surgery and ${ }^{5}$ Surgery, Division of Trauma and General Surgery, University of Pittsburgh Medical Center, Pittsburgh, Pennsylvania
\end{abstract}

\begin{abstract}
OBJECTIVE Blunt cerebrovascular injuries (BCVIs) following trauma carry risk for morbidity and mortality. Since patients with $\mathrm{BCVI}$ are often asymptomatic at presentation and neurological sequelae often occur within 72 hours, timely diagnosis is essential. Multidetector CT angiography (CTA) has been shown to be a noninvasive, cost-effective, reliable means of screening; however, the false-positive rate of CTA in diagnosing patients with BCVI represents a key drawback. Therefore, the authors assessed the role of DSA in the screening of BCVI when utilizing CTA as the initial screening modality.
\end{abstract}

METHODS The authors performed a retrospective analysis of patients who experienced BCVI between 2013 and 2015 at 2 Level I trauma centers. All patients underwent CTA screening for BCVI according to the updated Denver Screening Criteria. Patients who were diagnosed with BCVI on CTA underwent confirmatory digital subtraction angiography (DSA). Patient demographics, screening indication, BCVI grade on CTA and DSA, and laboratory values were collected. Comparison of false-positive rates stratified by BCVI grade on CTA was performed using the chi-square test.

RESULTS A total of 140 patients (64\% males, mean age 50 years) with 156 cerebrovascular blunt injuries to the carotid and/or vertebral arteries were identified. After comparison with DSA findings, CTA findings were incorrect in $61.5 \%$ of vessels studied, and the overall CTA false-positive rates were $47.4 \%$ of vessels studied and $47.9 \%$ of patients screened. The positive predictive value (PPV) for CTA was higher among worse BCVI subtypes on initial imaging (PPV 76\% and 97\%, for BCVI Grades II and IV, respectively) compared with Grade I injuries (PPV 30\%, p < 0.001).

CONCLUSIONS In the current series, multidetector CTA as a screening test for blunt cerebrovascular injury had a highfalse positive rate, especially in patients with Grade I BCVI. Given a false-positive rate of $47.9 \%$ with an estimated average of 132 patients per year screening positive for BCVI with CTA, approximately 63 patients per year would potentially be treated unnecessarily with antithrombotic therapy at a busy United States Level I trauma center. The authors' data support the use of DSA after positive findings on CTA in patients with suspected BCVI. DSA as an adjunctive test in patients with positive CTA findings allows for increased diagnostic accuracy in correctly diagnosing BCVI while minimizing risk from unnecessary antithrombotic therapy in polytrauma patients.

https://thejns.org/doi/abs/10.3171/2017.2.JNS163264

KEY WORDS blunt cerebrovascular injury; computed tomography angiography; digital subtraction angiography; trauma

$\mathrm{B}$ LUNT cerebrovascular injury (BCVI) is defined by the presence of damage to the carotid arteries (CAs) or vertebral arteries (VAs) as a result of nonpenetrating trauma. Early studies estimated the incidence of BCVI at roughly $0.1 \%$ among trauma patients. ${ }^{11}$ However, with the advent of more rigorous screening protocols and improved imaging modalities, recent studies have esti-

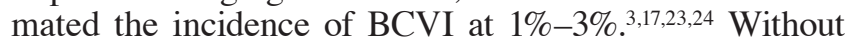
proper diagnosis and treatment, the estimated incidence of stroke in patients with VA and CA injuries is $10 \%-15 \%$

ABBREVIATIONS BCVI = blunt cerebrovascular injury; $C A=$ carotid artery; $C C A=$ common $C A ; C T A=C T$ angiography; $D S A=$ digital subtraction angiography; ICA = internal $C A ; P P V=$ positive predictive value; $V A=$ vertebral artery.

SUBMITTED December 29, 2016. ACCEPTED February 15, 2017.

INCLUDE WHEN CITING Published online August 11, 2017; DOI: 10.3171/2017.2.JNS163264. 
and $30 \%-40 \%$, respectively,,$^{4,9,10,15,20,21,24}$ with mortality of up to $40 \% .^{11,14}$

The 2 most commonly cited references regarding BCVI guidelines are from the Eastern and Western Trauma Associations. ${ }^{1,5}$ Both groups support the initial use of CT angiography (CTA) for screening and state that the findings obtained using this modality may be equivalent to those using digital subtraction angiography (DSA). While recent evidence sheds light on the low sensitivity $(51 \%-68 \%)$ and a high false-negative rate $(8 \%-24 \%)$ of CTA, much less attention has been paid to the high false-positive rate, where, in the most recently published data, up to $45 \%$ of patients are given a false diagnosis. ${ }^{12,21,22}$ This is of particular importance when considering the potential for iatrogenic hemorrhagic complications associated with the initiation of antithrombotic therapy in polytrauma patients. If the appropriateness of imaging recommendations depends on the adequacy of the screening modality, then CTA may render the aforementioned guidelines moot. In light of these concerns, and with the encouragement of the Western Trauma Association to formulate a local protocol, we initiated a guideline whereby patients with positive CTA findings underwent DSA as a confirmatory test.

\section{Methods}

We retrospectively reviewed all patients 18 years and older who had been admitted to the University of Pittsburgh Medical Center Presbyterian Hospital or University of Texas Health Science Center at San Antonio University Hospital, both Level I trauma centers, between June 2013 and November 2015, with BCVI diagnosed on screening CTA. The institutional review boards of both institutions approved this study. According to the updated Denver Screening Criteria protocol, patients were screened for BCVI based on their concomitant traumatic injuries on presentation as well as signs/symptoms consistent with BCVI. ${ }^{6}$ CTA of the head and neck was performed using a 64-channel, multidetector CT scanner, and all CT angiograms were read by board-certified neuroradiologists. In all patients diagnosed with BCVI based on CTA findings, DSA was performed within 24 hours of admission and images were read by a fellowship-trained interventionalist who was either a neurosurgeon or neurologist. Antithrombotic therapy was initiated based on initial CTA findings after clearance and discussion by the trauma and neurosurgery services. Given the current recommendations of the Eastern and Western Trauma Associations, which state that "the optimal treatment modality for the treatment of BCVI is as yet undetermined" and that "long-term antiplatelet therapy is preferable to warfarin for its safety and cost profile," in general, our preference was to use antiplatelet medications, namely, $325 \mathrm{mg}$ of aspirin daily, for the treatment of BCVI. We reserved anticoagulation, in the form of a nonbolus intravenous heparin drip followed by transition to oral warfarin, for patients with flow-limiting $(>75 \%)$ dissections or a significant amount of associated thrombus as visualized on CTA. In patients without evidence of BCVI on DSA, antithrombotic medications were stopped. In patients in whom catheter angiography confirmed the presence of vessel dissection, our protocol in- cluded 3 months of medical treatment with antithrombotic medication followed by repeat CTA. Major complications from DSA were defined as access site hematoma requiring blood transfusion, symptomatic vessel dissection/injury, new postprocedural neurological deficit/stroke, and acute kidney injury secondary to contrast-induced nephropathy. All patient information was de-identified and analyzed in compliance with Health Insurance Portability and Accountability Act regulations.

Baseline demographic and admission clinical data included age, sex, indication for BCVI screening, and BCVI grade on the initial CTA scan. ${ }^{2}$ The BCVI grades are as follows: Grade I, mild intimal injury or irregular intima; Grade II, dissection with raised intimal flap/intramural hematoma with luminal narrowing greater than $25 \%$; Grade III, pseudoaneurysm; Grade IV, vessel occlusion/thrombosis; and Grade V, vessel transection. Documentation of a true BCVI was identified through review of subsequent DSA studies and confirmed by radiology reports noting the presence of vascular injury as well as injury grade. In addition, serum creatinine levels were noted before and after CTA and DSA. Missing data were treated as omitted at random. The paired t-test was used to compare creatinine levels before and after CTA and DSA as well as for comparisons of BCVI grades based on CTA versus DSA results in the same patient. The positive predictive value (PPV) of CTA was defined as 1 minus the proportion of DSA Grade 0 (normal imaging findings with no injury) when the CTA grade was I or higher. The false-positive rates were compared for different CTA grades using the chi-square test. Significance was defined a priori as a $\mathrm{p}<$ 0.05 . Analyses were performed using $\mathrm{R}$ software (version 3, GNU Affero General Public License).

\section{Results}

The patient population comprised 140 patients $(64 \%$ males [89/140], mean age 50 years). The most common indication for BCVI screening was the presence of a cervical spine injury (67\%). The study cohort presented with a total of 156 BCVIs to the carotid and/or VAs. Of these patients, 127 sustained an injury to a single vessel, 10 suffered injuries to 2 vessels, and 3 presented with injuries to 3 vessels. Based on CTA evaluation, there were 2 injuries to the CCA, 45 injuries to the ICAs, and 109 injuries to the VAs (Table 1).

Based on confirmatory DSA, the most frequently observed vessel injuries observed in our cohort were VA injuries (63/82), with 27 dissections (BCVI Grades I and II; 33\%), 5 pseudoaneurysms (BCVI Grade III; 6\%), 30 occlusions (BCVI Grade IV; 37\%), and 1 transection (Grade V). The mean CTA BCVI grade was 1.92 , which was significantly higher than the mean DSA BCVI grade of $1.44(\mathrm{p}<0.001)$. DSA confirmed the same grade BCVI as CTA in $38.5 \%(60 / 156)$ of vessels studied; however, in $51.3 \%(80 / 156)$, the DSA grade was lower than that found on CTA, and in $10.3 \%(16 / 156)$, the DSA grade was demonstrated to be higher than that for initial CTA (Fig. 1).

The CTA false-positive rate (positive CTA findings but negative DSA findings) was $47.4 \%$ of vessels studied (47.9\% of patients screened), resulting in an overall PPV 
TABLE 1. Indications for BCVI screening and observed vessel injury on CTA

\begin{tabular}{lc}
\hline \multicolumn{1}{c}{ Indication } & No. of Vessels \\
\hline Traumatic injury/indication for BCVI screening & \\
\hline Cervical spine injury & 104 \\
\hline Closed head injury w/ DAI \& GCS score <6 & 5 \\
\hline Complex skull fracture & 29 \\
\hline Focal neurological deficit & 4 \\
\hline Mandible fracture & 2 \\
\hline Midface fracture/Le Fort II or III fracture & 8 \\
\hline Seat belt abrasion w/ pain or swelling & 4 \\
\hline Vessel injury on CTA & 2 \\
\hline CCA & 45 \\
\hline ICA & 109 \\
\hline VA
\end{tabular}

DAI = diffuse axonal injury; GCS = Glasgow Coma Scale.

of 53\% (82/156; Table 2). The difference in false-positive rates between the 2 centers included in our study was minimal: $47 \%$ of CTA studies performed at the University of Pittsburgh Presbyterian Hospital were proven to have false-positive findings based on confirmatory DSA; the false-positive rate for CTA studies at the University of Texas San Antonio Health Science Center University Hospital was $50 \%$. The PPV of CTA was higher for patients diagnosed with Grade II and Grade IV injuries (PPV 76\% and $97 \%$, respectively) compared with those with Grade I injuries (PPV 30\%, p < 0.001; Fig. 1 and Table 2). In addition, there was a significant difference between the PPV of BCVI found on screening CTA based on the vessel involved, with CTA demonstrating a PPV of $40.4 \%$ for injuries involving the CCA/ICAs and 58\% for injuries involving the VAs (Table 3). In particular, there were no instances of improper diagnosis of BCVI in cases in which VA occlusions were seen on CTA. Finally, the concomitant traumatic injury representing the indication for BCVI screening was a predictor $(p=0.004)$ of the PPV of BCVI found on CTA (Table 4); vessel injuries on CTA secondary to complex skull fractures demonstrated a PPV of only $28 \%$ after confirmatory DSA, whereas CTA showed a PPV of $60 \%$ for injuries diagnosed in association with cervical spine injuries.

The mean creatinine level before CTA was $0.86 \mathrm{mg} /$ dl (range $0.4-1.4 \mathrm{mg} / \mathrm{dl}$ ), which was significantly higher than the creatinine levels after CTA $(0.82 \mathrm{mg} / \mathrm{dl}$ [range

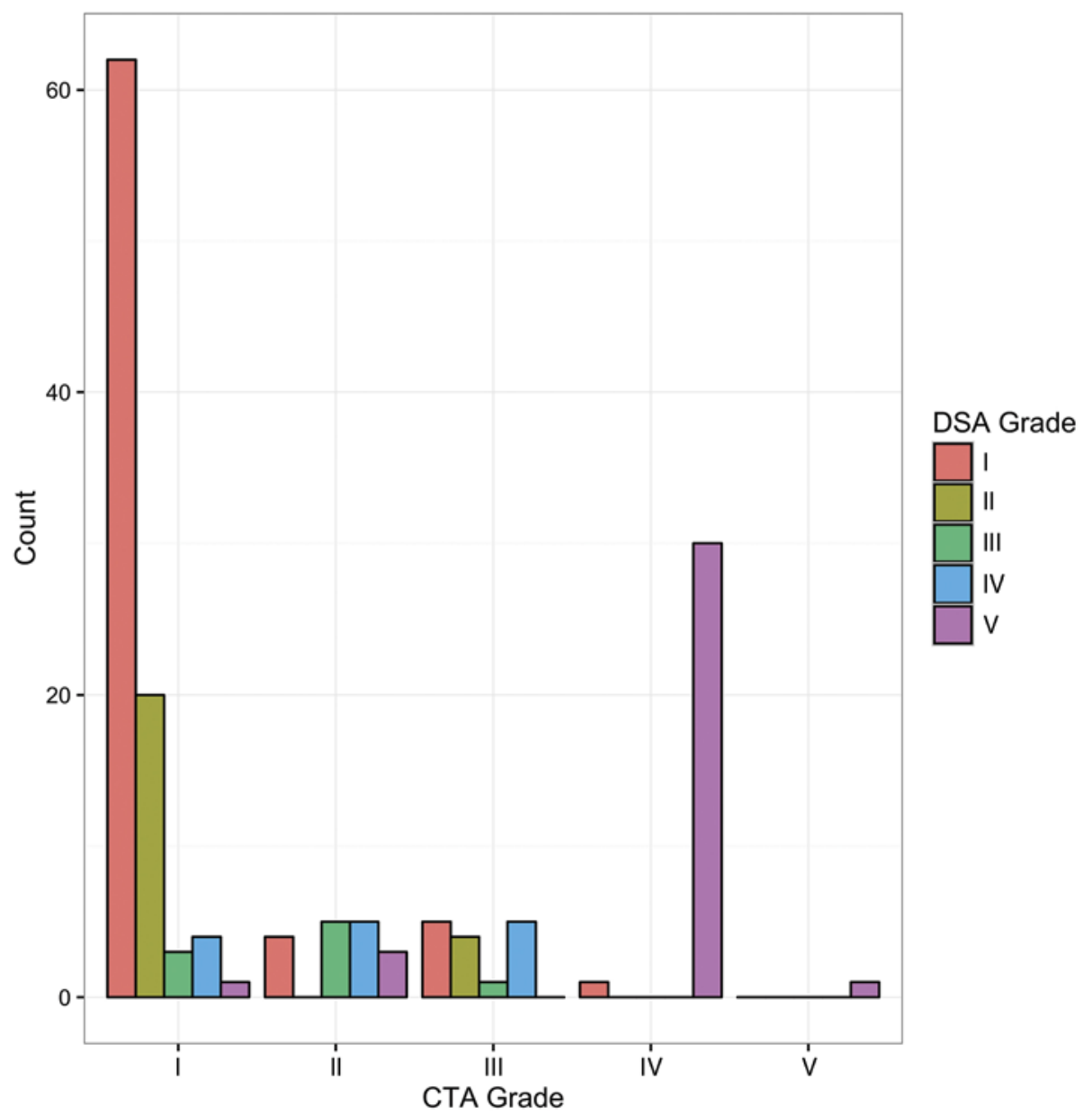

FIG. 1. Chart of study population comparing BCVI grade based on screening CTA versus BCVI grade on confirmatory DSA. Figure is available in color online only. 
TABLE 2. Accuracy of CTA based on grade of BCVI

\begin{tabular}{cccc}
\hline & \multicolumn{3}{c}{ No. of Vessels (\%) } \\
\cline { 2 - 4 } BCVI Grade & Total & False Positive & True Positive \\
\hline I & 92 & $64(69.57)$ & $28(30.43)$ \\
\hline II & 17 & $4(23.53)$ & $13(76.47)$ \\
\hline III & 15 & $5(33.33)$ & $10(66.67)$ \\
\hline IV & 31 & $1(3.23)$ & $30(96.77)$ \\
\hline V & 1 & $0(0)$ & $1(100)$ \\
\hline Total & 156 & $74(47.4)$ & $82(52.6)$ \\
\hline
\end{tabular}

0.29-1.4 mg/dl], $\mathrm{p}=0.001)$. The creatinine level following DSA was $0.76 \mathrm{mg} / \mathrm{dl}$ ( $\mathrm{n}=116$, range $0.19-1.4 \mathrm{mg} / \mathrm{dl})$. No major complications, including stroke, groin hematoma requiring blood transfusion, femoral pseudoaneurysm, or acute kidney injury, were caused by the DSA.

\section{Discussion}

CTA remains the primary screening modality for BCVI because of its speed, availability, noninvasive nature, safety, relative efficacy, and low cost. Current guidelines support its use; however, the guidelines depend on the sensitivity and specificity of the screening modality. The high false-negative rates reported by other centers call these guidelines into question. The high false-positive rates presented in this paper further support a change to existing guidelines.

In the current study, we found that CTA provided an incorrect BCVI grade in $61.5 \%$ of vessels studied with a false-positive rate of $47 \%$ among our patient population when compared with DSA. The PPV of CTA was increased for Grade II and Grade IV injuries, compared with Grade I injuries, demonstrating that CTA is more likely to correctly diagnose more severe vascular injuries. In addition, we identified CCA and ICA injuries and the presence of complex skull fractures as particular risk factors for false-positive CTA results. Regarding the cited complica-

\section{TABLE 3. Accuracy of CTA based on location and severity of} BCVI

\begin{tabular}{lrcc}
\hline \multicolumn{1}{c}{ Vessel Injury } & Total & False Positive $(\%)$ & True Positive (\%) \\
\hline Carotid artery & & & \\
\hline CCA dissection & 2 & $2(100)$ & $0(0)$ \\
\hline ICA dissection & 35 & $21(60)$ & $14(40)$ \\
\hline ICA pseudoaneurysm & 9 & $4(44)$ & $5(56)$ \\
\hline ICA occlusion & 1 & $1(100)$ & $0(0)$ \\
\hline Total & 47 & $28(59.6)$ & $19(40.4)$ \\
\hline VA & & & \\
\hline VA dissection & 72 & $45(62.5)$ & $27(37.5)$ \\
\hline VA occlusion & 30 & $0(0)$ & $30(100)$ \\
\hline VA pseudoaneurysm & 6 & $1(16.7)$ & $5(83.3)$ \\
\hline VA transection & 1 & $0(0)$ & $1(100)$ \\
\hline Total & 109 & $46(42.2)$ & $63(57.8)$ \\
\hline
\end{tabular}

TABLE 4. Accuracy of CTA based on indication for BCVI

\begin{tabular}{lrcc}
\hline \multirow{1}{*}{$\begin{array}{c}\text { Indication for } \\
\text { BCVI Screening }\end{array}$} & \multicolumn{3}{c}{ No. of Vessels (\%) } \\
\cline { 2 - 4 } & Total & False Positive & True Positive \\
\hline Cervical spine injury & 104 & $42(40.38)$ & $62(59.62)$ \\
\hline $\begin{array}{l}\text { Closed head injury w/ DAI } \\
\quad \text { \& GCS score <6 }\end{array}$ & 5 & $1(20)$ & $4(80)$ \\
\hline Complex skull fracture & 29 & $21(72.41)$ & $8(27.59)$ \\
\hline $\begin{array}{l}\text { Focal neurological deficit } \\
\text { after TBI }\end{array}$ & 4 & $2(50)$ & $2(50)$ \\
\hline Mandible fracture & 2 & $2(100)$ & $0(0)$ \\
\hline Midface fracture & 8 & $5(62.5)$ & $3(37.5)$ \\
\hline Seat belt abrasion w/ pain & 4 & $1(25)$ & $3(75)$ \\
\hline Total & 156 & $74(47.4)$ & $82(52.6)$ \\
\hline
\end{tabular}

$\mathrm{TBI}=$ traumatic brain injury.

tions associated with DSA, we observed none. There were no associated strokes. Serum creatinine levels following both CTA and follow-up DSA were, in fact, lower than on initial patient presentation to the trauma bay. While DSA risks are not zero, complications at high-volume centers are low, particularly in young, otherwise healthy, trauma patients.

The utility of performing confirmatory DSA in patients with positive findings on CTA is especially important in the trauma population, in which many individuals present with multisystem injuries. In the experience of Stein and colleagues at the R. Adams Cowley Shock Trauma Center at the University of Maryland School of Medicine, ${ }^{16}$ nearly $25 \%$ of patients with BCVI were not treated with antithrombotic medications due to concomitant injuries, the most common of which were traumatic brain injury, spinal cord injury, ${ }^{16}$ visceral injury, multiple orthopedic injuries, or early mortality. Furthermore, among the individuals who required antithrombotic medications, initiation of therapy was delayed beyond 48 hours in nearly $50 \%$ of patients due to traumatic brain injury or imminent surgery. Studies examining the hemorrhagic complications associated with the administration of antithrombotic medications in polytrauma patients with BCVI have yielded conflicting results. No significant difference in hemorrhagic complications and/or safety of initiation of antithrombotic therapy was documented in 3 separate analyses involving institutional reviews. ${ }^{6,7,22}$ However, a criticism of 2 of the studies is that either patients were not actually started on a regimen of antithrombotic therapy soon after presentation or that the actual time to initiation of therapy was not documented. Other studies have demonstrated serious bleeding complications associated with antithrombotic therapy for the treatment of BCVI, specifically noting increased hemorrhagic events in patients with suspected BCVI who were treated with anticoagulants. These increased rates of hemorrhage span the gamut from $8 \%-22 \%$ to over $50 \%$. $^{3,4,13,18-20,25}$

If the false-positive rates of CTA are as high as $47 \%$, it is possible that these patients may have been treated for an injury they did not have and derived a complication from a therapy they did not need. In addition, finding no injury on DSA obviates the need for follow-up imaging and out- 
TABLE 5. Change in clinical management based on confirmatory DSA

\begin{tabular}{lc}
\hline \multicolumn{1}{c}{ Variable } & No. of Vessels $(\%)$ \\
\hline Positive CTA w/ negative DSA & $74(47.4)$ \\
\hline Anticoagulation initiated after DSA & $8(5.13)$ \\
\hline Intervention w/ stenting during DSA & $5(3.21)$ \\
\hline Total & $87(55.77)$ \\
\hline
\end{tabular}

patient appointments, which minimizes the waste of important resources. A recent publication evaluated a similar protocol of performing confirmatory DSA in patients with a positive CTA result and yielded a similar false-positive rate of $45 \%$, as was found in our analysis. ${ }^{22}$ The authors started all patients on a regimen of therapeutic anticoagulation with intravenous heparin, aiming for a partial thromboplastin time of 40-50 seconds based on the diagnosis of BCVI by CTA until DSA could be performed. After the confirmatory DSA, patients continued to receive anticoagulation therapy or were transitioned to antiplatelet therapy based on the vessel involved and injury grade. As we found in our cohort, Shahan et al. found that the vast majority $(87 \%)$ of false-positive CTA results were associated with Grade I injuries on CTA; however, in contrast to our findings, they noted that VA injuries were more commonly misdiagnosed $(57 \%){ }^{22}$

In summary, our study has demonstrated that CTA provides an incorrect BCVI grade in $61.5 \%$ of cases and has a high false-positive rate of $47.4 \%$ (Table 5). Taken in context with the recently published series by Shahan et al., our findings from 2 different centers confirm that false-positive CTA findings in the setting of BCVI are not isolated to a single group of neuroradiologists. ${ }^{22}$ In addition, patients presenting with complex skull fractures were more likely to have false-positive CTA findings than those with cervical spine injuries.

Limitations of this study include its retrospective nature, which inhibited our collection of information, such as hemorrhagic complications from anticoagulation. Other limitations include the application of DSA only in patients with positive CTA findings, thereby inhibiting the calculation of false negatives. Finally, further study is required to determine the reason for our high rate of false-negative readings. For instance, we noted a number of indeterminate or "hedge" readings that were ultimately considered positive CTA findings. This subset requires further attention from our neuroradiologists.

\section{Conclusions}

The current findings support the safety and utility of DSA as a confirmatory test to be used in conjunction with CTA in patients with positive, or abnormal, results. To that end, given a false-positive rate of $47.9 \%$ with an estimated average of 132 patients per year with positive findings for BCVI with CTA, approximately 63 patients per year would potentially be treated unnecessarily with antithrombotic therapy at a busy United States Level I trauma center. These data also highlight the need for a prospective trial in a larger patient population with at least 2 blinded CTA in- terpretations performed by those not performing the DSA. Future goals will be to determine the subset of patients who would not require confirmatory DSA, to determine the interobserver variability in CTA interpretation, to elucidate the cause of false-positive radiographic readings, and to contribute to future BCVI screening guidelines, incorporating judicious use of confirmatory DSA.

\section{References}

1. Biffl WL, Cothren CC, Moore EE, Kozar R, Cocanour C, Davis JW, et al: Western Trauma Association critical decisions in trauma: screening for and treatment of blunt cerebrovascular injuries. J Trauma 67:1150-1153, 2009

2. Biffl WL, Moore EE, Offner PJ, Brega KE, Franciose RJ, Burch JM: Blunt carotid arterial injuries: implications of a new grading scale. J Trauma 47:845-853, 1999

3. Biffl WL, Moore EE, Ryu RK, Offner PJ, Novak Z, Coldwell DM, et al: The unrecognized epidemic of blunt carotid arterial injuries: early diagnosis improves neurologic outcome. Ann Surg 228:462-470, 1998

4. Biffl WL, Ray CE Jr, Moore EE, Franciose RJ, Aly S, Heyrosa MG, et al: Treatment-related outcomes from blunt cerebrovascular injuries: importance of routine follow-up arteriography. Ann Surg 235:699-707, 2002

5. Bromberg WJ, Collier BC, Diebel LN, Dwyer KM, Holevar MR, Jacobs DG, et al: Blunt cerebrovascular injury practice management guidelines: the Eastern Association for the Surgery of Trauma. J Trauma 68:471-477, 2010

6. Burlen CC, Biffl WL, Moore EE, Barnett CC, Johnson JL, Bensard DD: Blunt cerebrovascular injuries: redefining screening criteria in the era of non-invasive diagnosis. J Trauma Acute Care Surg 72:330-337, 2012

7. Byrnes MC, Irwin E, Roach R, James M, Horst PK, Reicks P: Therapeutic anticoagulation can be safely accomplished in selected patients with traumatic intracranial hemorrhage. World J Emerg Surg 7:25, 2012

8. Callcut RA, Hanseman DJ, Solan PD, Kadon KS, Ingalls NK, Fortuna GR, et al: Early treatment of blunt cerebrovascular injury with concomitant hemorrhagic neurologic injury is safe and effective. J Trauma Acute Care Surg 72:338346,2012

9. Cothren CC, Biffl WL, Moore EE, Kashuk JL, Johnson JL: Treatment for blunt cerebrovascular injuries: equivalence of anticoagulation and antiplatelet agents. Arch Surg 144:685690, 2009

10. Cothren CC, Moore EE: Blunt cerebrovascular injuries. Clinics (Sao Paulo) 60:489-496, 2005

11. Davis JW, Holbrook TL, Hoyt DB, Mackersie RC, Field TO Jr, Shackford SR: Blunt carotid artery dissection: incidence, associated injuries, screening, and treatment. J Trauma 30:1514-1517, 1990

12. DiCocco JM, Emmett KP, Fabian TC, Zarzaur BL, Williams JS, Croce MA: Blunt cerebrovascular injury screening with 32-channel multidetector computed tomography: more slices still don't cut it. Ann Surg 253:444-450, 2011

13. Eachempati SR, Vaslef SN, Sebastian MW, Reed RL II: Blunt vascular injuries of the head and neck: is heparinization necessary? J Trauma 45:997-1004, 1998

14. Eastham S: Blunt cerebrovascular injuries in trauma. Int J Surg 33(Pt B):251-253, 2016

15. Emmett KP, Fabian TC, DiCocco JM, Zarzaur BL, Croce MA: Improving the screening criteria for blunt cerebrovascular injury: the appropriate role for computed tomography angiography. J Trauma 70:1058-1065, 2011

16. Goodwin RB, Beery PR II, Dorbish RJ, Betz JA, Hari JK, Opalek JM, et al: Computed tomographic angiography versus conventional angiography for the diagnosis of blunt cerebro- 
vascular injury in trauma patients. J Trauma 67:1046-1050, 2009

17. Hughes KM, Collier B, Greene KA, Kurek S: Traumatic carotid artery dissection: a significant incidental finding. Am Surg 66:1023-1027, 2000

18. Kerwin AJ, Bynoe RP, Murray J, Hudson ER, Close TP, Gifford RR, et al: Liberalized screening for blunt carotid and vertebral artery injuries is justified. J Trauma 51:308-314, 2001

19. Miller PR, Fabian TC, Bee TK, Timmons S, Chamsuddin A, Finkle R, et al: Blunt cerebrovascular injuries: diagnosis and treatment. J Trauma 51:279-286, 2001

20. Miller PR, Fabian TC, Croce MA, Cagiannos C, Williams JS, Vang M, et al: Prospective screening for blunt cerebrovascular injuries: analysis of diagnostic modalities and outcomes. Ann Surg 236:386-395, 2002

21. Paulus EM, Fabian TC, Savage SA, Zarzaur BL, Botta V, Dutton W, et al: Blunt cerebrovascular injury screening with 64-channel multidetector computed tomography: more slices finally cut it. J Trauma Acute Care Surg 76:279-285, 2014

22. Shahan CP, Magnotti LJ, Stickley SM, Weinberg JA, Hendrick LE, Uhlmann RA, et al: A safe and effective management strategy for blunt cerebrovascular injury: Avoiding unnecessary anticoagulation and eliminating stroke. J Trauma Acute Care Surg 80:915-922, 2016

23. Sliker CW, Shanmuganathan K, Mirvis SE: Diagnosis of blunt cerebrovascular injuries with 16-MDCT: accuracy of whole-body MDCT compared with neck MDCT angiography. AJR Am J Roentgenol 190:790-799, 2008
24. Stein DM, Boswell S, Sliker CW, Lui FY, Scalea TM: Blunt cerebrovascular injuries: does treatment always matter? J Trauma 66:132-144, 2009

25. Wahl WL, Brandt MM, Thompson BG, Taheri PA, Greenfield LJ: Antiplatelet therapy: an alternative to heparin for blunt carotid injury. J Trauma 52:896-901, 2002

\section{Disclosures}

The authors report no conflict of interest concerning the materials or methods used in this study or the findings specified in this paper.

\section{Author Contributions}

Conception and design: Jankowitz, Grandhi, Weiner, Agarwal, Okonkwo. Acquisition of data: Grandhi, Weiner, Agarwal. Analysis and interpretation of data: Jankowitz, Grandhi, Weiner, Agarwal, Panczykowski, Ares, Gelfond, Okonkwo. Drafting the article: Jankowitz, Grandhi, Weiner, Agarwal, Rodriguez, Okonkwo. Critically revising the article: all authors. Reviewed submitted version of manuscript: all authors. Statistical analysis: Gelfond. Administrative/technical/material support: Jankowitz. Study supervision: Jankowitz.

\section{Correspondence}

Brian T. Jankowitz, Department of Neurological Surgery, University of Pittsburgh Medical Center, 1350 Locust St., Ste. 300, MPOB, Pittsburgh, PA 15219. email: jankbt@upmc.edu. 